\title{
CANTOR BOUQUETS, EXPLOSIONS, AND KNASTER CONTINUA: DYNAMICS OF COMPLEX EXPONENTIALS
}

\author{
Robert L. Devaney
}

\begin{abstract}
We describe some of the interesting dynamical and topological properties of the complex exponential family $\lambda e^{z}$ and its associated Julia sets.
\end{abstract}

\section{Introduction}

Our goal in this paper is to describe some of the topology and dynamics of the complex exponential family $E_{\lambda}(z)=\lambda e^{z}$. We will restrict to $\lambda$-values that are real and positive, mainly because all of the interesting phenomena present for other complex $\lambda$-values is already present in this situation.

For a complex analytic function $E$, the interesting orbits lie in the Julia set, which we denote by $J(E)$. For the exponential family, the Julia set of $E_{\lambda}$ has three characterizations:

1. $J\left(E_{\lambda}\right)$ is the set of points at which the family of iterates of $E_{\lambda}$, $\left\{E_{\lambda}^{n}\right\}$ is not a normal family in the sense of Montel.

2. $J\left(E_{\lambda}\right)$ is the closure of the set of repelling periodic points of $E_{\lambda}$.

3. $J\left(E_{\lambda}\right)$ is the closure of the set of points whose orbits tend to $\infty$.

Note that condition 3 differs markedly from the case of polynomial iterations, where $J(E)$ is the boundary of the set of escaping orbits. The reason for the difference is $E_{\lambda}$ has an essential singularity at $\infty$, while polynomials have superattracting fixed points at $\infty$. The equivalence of 1 and 2 was shown by Baker, see [Ba2]. The equivalence of 1 and 3 is shown in $[\mathbf{D T}]$. The complement of the Julia set is called the stable set.

In $J\left(E_{\lambda}\right)$, there are two very interesting topological structures, Cantor bouquets and Knaster-like continua. We will describe the construction of each in detail. 
The Julia set for $E_{\lambda}$ undergoes a remarkable transformation as $\lambda$ passes through $1 / e$. We will show in Section 1 that $J\left(E_{\lambda}\right)$ is a Cantor bouquet for $0<\lambda \leq 1 / e$. Roughly speaking, a Cantor bouquet has the property that all points in the set lie on a curve homeomorphic to a closed half line. Each of these curves in $J\left(E_{\lambda}\right)$ extend to $\infty$ in the right half-plane. All repelling periodic points and points with bounded orbits lie at the endpoints of the curves, while points that do not lie at the endpoints have unbounded orbits. Since repelling periodic points are dense in $J\left(E_{\lambda}\right)$, the endpoints of the Cantor bouquet must be dense in $J\left(E_{\lambda}\right)$. Indeed, we will show that the set of endpoints is a totally disconnected set, but that the set of endpoints together with the point at infinity forms a connected set.

At $\lambda=1 / e, E_{\lambda}$ undergoes a simple saddle-node bifurcation. An attracting fixed point merges with a repelling fixed point at this $\lambda$-value, producing a neutral fixed point. When $\lambda>1 / e$, this neutral fixed point gives way to a pair of repelling fixed points.

This apparently simple bifurcation has profound global ramifications. When $\lambda \leq 1 / e$, we will show that the Cantor bouquet that forms the Julia set is a nowhere dense subset of the right half plane. However, when $\lambda>1 / e, J\left(E_{\lambda}\right)$ suddenly becomes the whole plane. No new repelling periodic points (except the two fixed points involved in the saddle-node) are born in this bifurcation; all simply move continuously as $\lambda$ crosses through $1 / e$. Yet somehow, as soon as $\lambda$ exceeds $1 / e$, the repelling periodic points become dense in $\mathbb{C}$.

At this bifurcation, the attracting fixed point and its entire basin of attraction disappear. Most of the structure of the Cantor bouquet remains in the Julia set. However, a new and interesting topological invariant set arises. We will show that this set is an indecomposable continuum on which most orbits cycle toward the orbit of 0 and $\infty$.

\section{Exponential Dynamics}

As in the often-studied quadratic family $Q_{c}(z)=z^{2}+c$, it is the orbit of 0 that plays a crucial role in determining the dynamics of $E_{\lambda}$. For the exponential family, 0 is an asymptotic value rather than a critical point. Nevertheless, any stable domain in the complement of the Julia set of $E_{\lambda}$ must be associated with the orbit of 0 in the following sense:

Theorem 2.1. Suppose $E_{\lambda}$ has an attracting or parabolic periodic point. Then $E_{\lambda}^{n}(0)$ must tend to the attracting or parabolic cycle. If, on the other hand, $E_{\lambda}^{n}(0) \rightarrow \infty$, then $J\left(E_{\lambda}\right)=\mathbb{C}$. 
The proof of the first statement in this theorem is a classical fact that goes back to Fatou. The second follows from the Sullivan No Wandering Domains Theorem [Su], as extended to the case of the exponential by Goldberg and Keen $[\mathbf{G K}]$ and Eremenko and Lyubich $[\mathbf{E L}]$. Rather than rely on this big machinery, we will give a bare-hands approach due to Misiurewicz $[\mathbf{M i}]$ to show that $J\left(E_{\lambda}\right)=\mathbb{C}$ when $\lambda>1 / e$ in Section 5 .

The exponential family undergoes a saddle node bifurcation at $\lambda=1 / e$ since, when $\lambda=1 / e$, the graph of $E_{1 / e}$ is tangent to the diagonal at 1 . See Figure 1 . We have $E_{1 / e}(1)=1$ and $E_{1 / e}^{\prime}(1)=1$. When $\lambda>1 / e$, the graph of $E_{\lambda}$ lies above the diagonal and all orbits (including 0 ) tend to $\infty$. When $\lambda<1 / e$, the graph of $E_{\lambda}$ crosses the diagonal twice, at an attracting fixed point $a_{\lambda}$ and a repeling fixed point $r_{\lambda}$. For later use note that $0<a_{\lambda}<1<r_{\lambda}$. Note also that the orbit of 0 tends to $a_{\lambda}$, as it must by Fatou's theorem. See Figure 1.

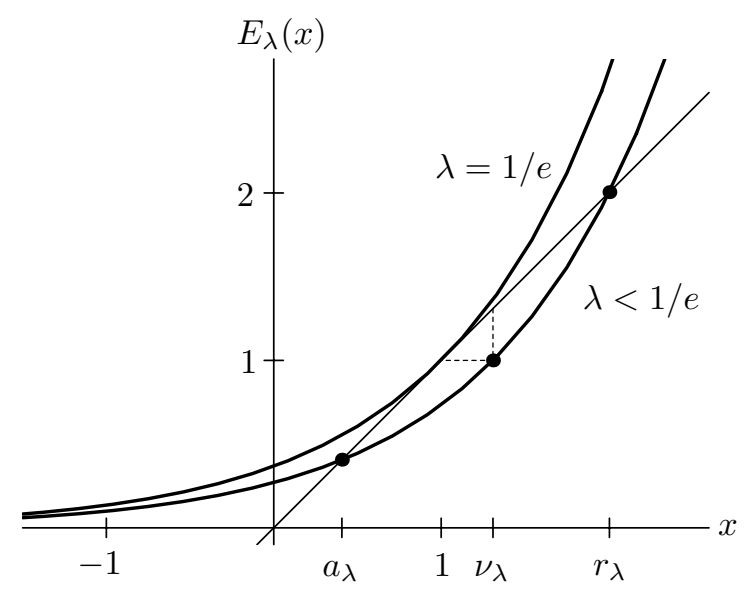

Figure 1. The graphs of $E_{\lambda}$ for $\lambda=1 / e$ and $\lambda<1 / e$.

\section{Cantor Bouquets}

In this section, we begin the study of the dynamics of $E_{\lambda}$ by considering the case where $\lambda \leq 1 / e$. We show here that $J\left(E_{\lambda}\right)$ is a Cantor bouquet.

In Figure 2, we display the Julia set for $E_{1 / e}$. The complement of the Julia set is displayed in black. It appears that this Julia set contains large open sets, but this in fact is not the case. The Julia set actually consists of uncountably many curves or "hairs" extending to $\infty$ in the right half plane. Each of the "fingers" in this figure seems to have many smaller fingers protruding from them. As we zoom in to this image, we see more and more of the self-similar structure, as each finger generates 
more and more fingers. In fact, each of these fingers consists of a cluster of hairs that are packed together so tightly that the resulting set has Hausdorff dimension 2.

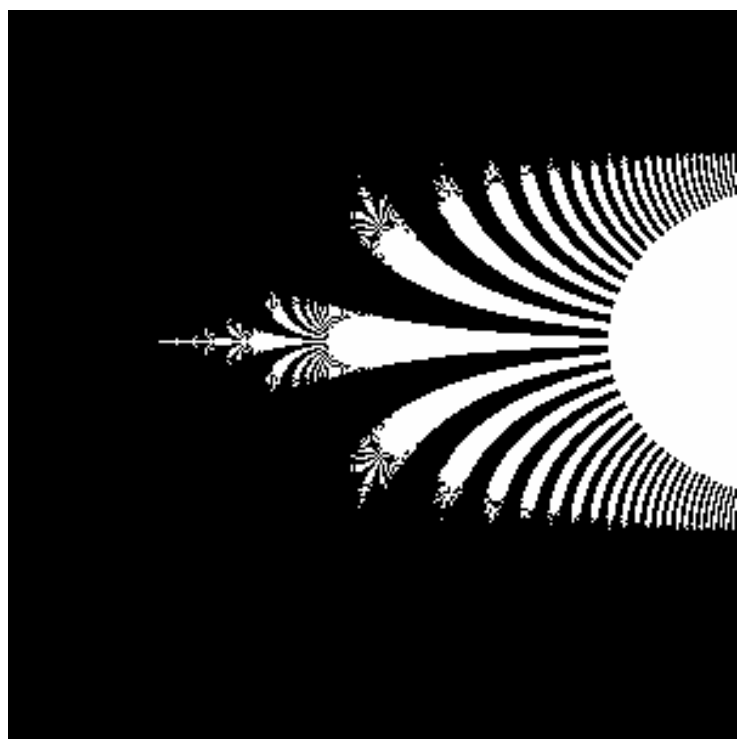

Figure 2. The Julia set for $\lambda=1 / e$.

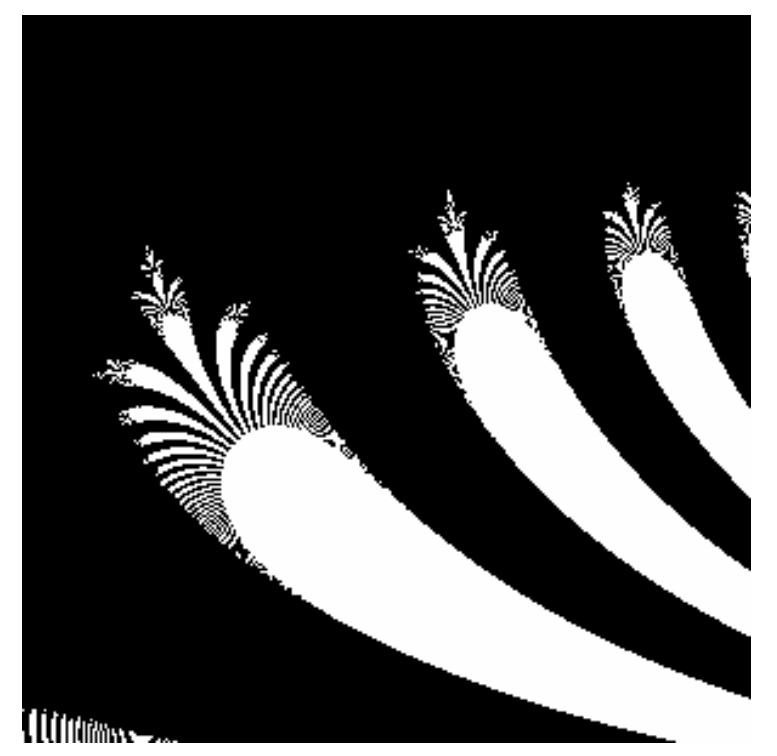

Figure 3. Magnification of the Julia set for $\lambda=1 / e$. 


\subsection{The Idea of the Construction.}

Here is a rough idea of the construction of a Cantor bouquet. We will "tighten up" these ideas in following sections.

Let $E(z)=(1 / e) e^{z}$. We have $E(1)=1$ and $E^{\prime}(1)=1$. If $x_{0} \in \mathbb{R}$ and $x<1$, then $E^{n}\left(x_{0}\right)$ tends to the fixed point at 1 . If $x_{0}>1$, then $E^{n}\left(x_{0}\right) \rightarrow \infty$ as $n \rightarrow \infty$. This can be shown using the web diagram as shown in Figure 4.

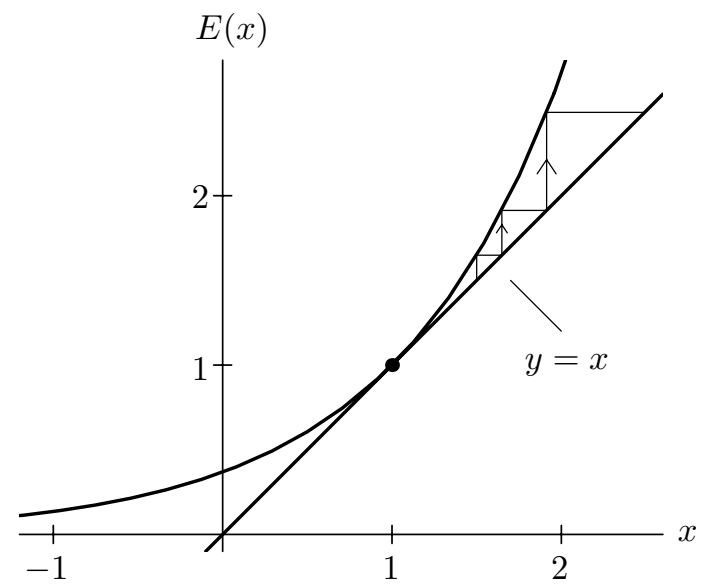

Figure 4. The graph of $E(x)=(1 / e) e^{x}$.

The vertical line $\operatorname{Re} z=1$ is mapped to the circle of radius 1 centered at the origin. In fact, $E$ is a contraction in the half plane $H$ to the left of this line, since

$$
\left|E^{\prime}(z)\right|=\frac{1}{e} \exp (\operatorname{Re} z)<1
$$

if $z \in H$. Consequently, all points in $H$ have orbits that tend to 1 . Hence this half plane lies in the stable set, i.e., in the complement of the Julia set. We will try to paint the picture of the Julia set of $E$ by painting instead its complement.

Since the half plane $H$ is forward invariant under $E$, we can obtain the entire stable set by considering all preimages of this half plane. Now the first preimage of $H$ certainly contains the horizontal $\operatorname{lines} \operatorname{Im} z=$ $(2 k+1) \pi, \operatorname{Re} z \geq 1$, for each integer $k$, since $E$ maps these lines to the negative real axis which lies in $H$. Hence there are open neighborhoods of each of these lines that lie in the stable set. The first preimage of $H$ is shown in Figure 5. The complement of $E^{-1}(H)$ consists of infinitely 
many "fingers". The fingers are $2 k \pi i$ translates of each other, and each is mapped onto the complementary half plane $\operatorname{Re} z \geq 1$.

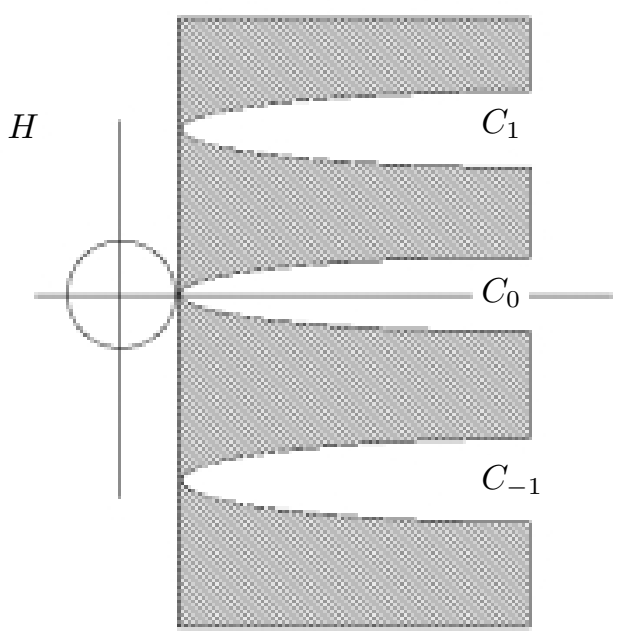

Figure 5. The preimage of $H$ consists of $H$ and the shaded region.

We denote the fingers in the complement of $E^{-1}(H)$ by $C_{j}$ with $j \in \mathbb{Z}$, where $C_{j}$ contains the half line $\operatorname{Im} z=2 j \pi, \operatorname{Re} z \geq 1$, which is mapped into the positive real axis. That is, the $C_{j}$ are indexed by the integers in order of increasing imaginary part. Note that $C_{j}$ is contained within the strip $-\frac{\pi}{2}+2 j \pi \leq \operatorname{Im} z \leq \frac{\pi}{2}+2 j \pi$.

Now each $C_{j}$ is mapped in one-to-one fashion onto the entire half plane $\operatorname{Re} z \geq 1$. Consequently each $C_{j}$ contains a preimage of each other $C_{k}$. Each of these preimages forms a subfinger which extends to the right. See Figure 6. The complement of these subfingers necessarily lies in the stable set.

Now we continue inductively. Each subfinger is mapped onto one of the original fingers by $E$. Consequently, there are infinitely many sub-subfingers which are mapped to the $C_{j}$ 's by $E^{2}$. So at each stage we remove the complement of infinitely many subfingers from each remaining finger.

This process is reminiscent of the construction of the Cantor set in the dynamics of polynomials when all critical points tend to $\infty$. In that construction, the complements of disks are removed at each stage; here we remove the complement of infinitely many fingers. As a result, after performing this operation infinitely many times, we do not end up with points. Rather, as we will see, the intersection of all of these fingers is a simple curve extending to $\infty$. 


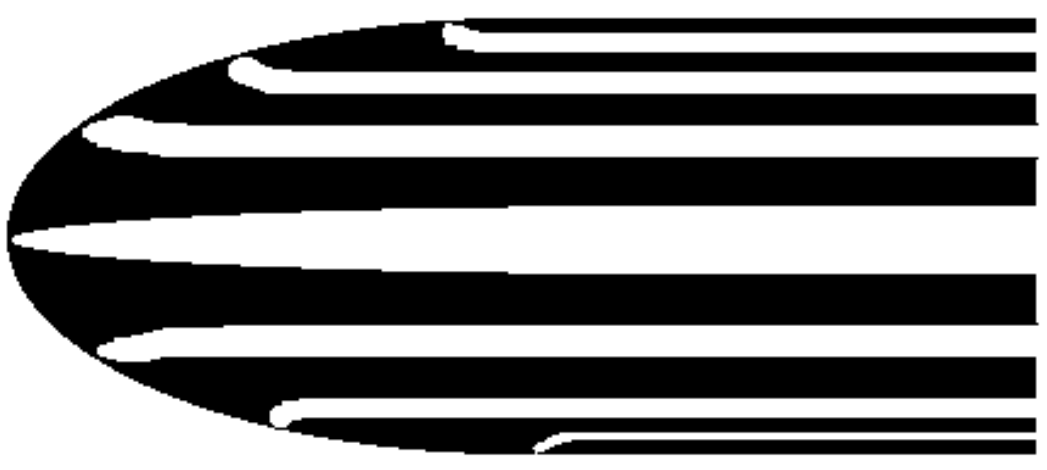

Figure 6 . The second preimage of $H$ in one of the fingers $C_{j}$.

This collection of curves forms the Julia set. $E$ permutes these curves and each curve consists of a well-defined endpoint together with a "hair" which extends to $\infty$. It is tempting to think of this structure as a "Cantor set of curves", i.e., a product of the set of endpoints and the half-line. However, this is not the case as the set of endpoints is not closed.

Note that we can assign symbolic sequences to each point on these curves. We simply watch which of the $C_{j}$ 's these orbit of the point lies in after each iteration and assign the corresponding index $j$. That is, to each hair in the Julia set we attach an infinite sequence $s_{0} s_{1} s_{2} \ldots$ where $s_{j} \in \mathbb{Z}$ and $s_{j}=k$ if the $j^{\text {th }}$ iterate of the hair lies in $C_{k}$. The sequence $s_{0} s_{1} s_{2} \ldots$ is called the itinerary of the curve.

For example, the portion of the real line $\{x \mid x \geq 1\}$ is an invariant curve in the Julia set since all points (except 1 ) tend to $\infty$ under iteration, not to the fixed point. These points all have itinerary $000 \ldots$.

One temptation is to say that there is a hair corresponding to every possible sequence $s_{0} s_{1} s_{2} \ldots$. This, unfortunately, is not true, as certain sequences simply grow too quickly to correspond to orbits of $E$.

So this is $J(E)$ : a "hairy" object extending toward $\infty$ in the righthalf plane. We call this object a Cantor bouquet. We will see that this bouquet has some rather interesting topological properties as we investigate further.

\subsection{Straight Brushes.}

To describe the structure of a Cantor bouquet, we need to introduce the notion of a straight brush. 
To each irrational number $\zeta$, we assign an infinite string of integers $n_{0} n_{1} n_{2} \ldots$ as follows. We will break up the real line into open intervals $I_{n_{0} n_{1} \ldots n_{k}}$ which have the following properties

1. $I_{n_{0} \ldots n_{k}}$ strictly contains $I_{n_{0} \ldots n_{k+1}}$.

2. The endpoints of $I_{n_{0} \ldots n_{k}}$ are rational.

3. $\zeta=\bigcap_{k=1}^{\infty} I_{n_{0} \ldots n_{k}}$.

Now there are many ways to do this. We choose the following inductive method based on the Farey tree. For any $k \in \mathbb{Z}$, we first define $I_{k}=$ $(k, k+1)$. Given $I_{n_{0} \ldots n_{k}}$ and $j \in \mathbb{Z}$, we define $I_{n_{0} \ldots n_{k} j}$ as follows. Suppose

$$
I_{n_{0} \ldots n_{k}}=\left(\frac{\alpha}{\beta}, \frac{\gamma}{\delta}\right)
$$

and let $p_{0} / q_{0}=(\alpha+\gamma) /(\beta+\delta)$, the Farey child of $\alpha / \beta$ and $\gamma / \delta$. Let $p_{n} / q_{n}$ be the Farey child of $p_{n-1} / q_{n-1}$ and $\gamma / \delta$ for $n>0$, and let $p_{n-1} / q_{n-1}$ be the Farey child for $\alpha / \beta$ and $p_{n} / q_{n}$ for $n \leq 0$. We then set $I_{n_{0} \ldots n_{k} j}$ to be the open interval $\left(p_{j} / q_{j}, p_{j+1} / q_{j+1}\right)$.

Example. $I_{0}=(0,1)$. The Farey child of $0 / 1$ and $1 / 1$ is $1 / 2$, so $p_{0} / q_{0}=1 / 2$. Then $p_{1} / q_{1}=\frac{1}{2} \oplus \frac{1}{1}=2 / 3, p_{2} / q_{2}=\frac{2}{3} \oplus \frac{1}{1}=\frac{3}{4}$, and $p_{n} / q_{n}=n+1 / n+2$ for $n>0$.

For the remaining $n$ we have

$$
\begin{aligned}
& p_{-1} / q_{-1}=\frac{0}{1} \oplus \frac{1}{2}=\frac{1}{3} \\
& p_{-2} / q_{-2}=\frac{0}{1} \oplus \frac{1}{3}=\frac{1}{4} \\
& p_{-n} / q_{-n}=\frac{1}{n+2} .
\end{aligned}
$$

Therefore, if $n \geq 0$,

$$
I_{0 n}=\left(\frac{n+1}{n+2}, \frac{n+2}{n+3}\right)
$$

and if $n<0$,

$$
I_{0 n}=\left(\frac{1}{-n+2}, \frac{1}{-n+1}\right) .
$$


See Figure 7. Note that we exhaust all of the rationals via this procedure, so each irrational is contained in a unique $I_{n_{0} n_{1} \ldots}$. Note that the endpoints of the successive intervals $I_{n_{0}}, I_{n_{0} n_{1}}, \ldots$ correspond to the usual sequence of convergents in the continued fraction expansion of $\xi$.

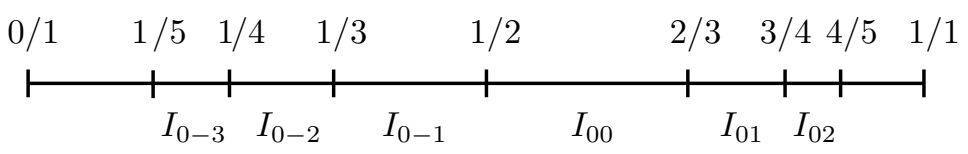

Figure 7. Construction of $I_{0 n}$.

We now define a straight brush, a notion due to Aarts and Oversteegen $[\mathbf{A O}]$.

Definition 3.1. A straight brush $B$ is a subset of $[0, \infty) \times \mathcal{N}$, where $\mathcal{N}$ is a dense subset of irrationals and $B$ has the following 3 properties.

1. $B$ is "hairy" in the following sense. If $(y, \alpha) \in B$, then there exists a $y_{\alpha} \leq y$ such that $(t, \alpha) \in B$ iff $t \geq y_{\alpha}$. That is, the "hair" $(t, \alpha)$ is contained in $B$ where $t \geq y_{\alpha} . y_{\alpha}$ is called the endpoint of the hair corresponding to $\alpha$.

2. Given an endpoint $\left(y_{\alpha}, \alpha\right) \in B$ there are sequences $\beta_{n} \nearrow \alpha$ and $\gamma_{n} \searrow \alpha$ in $\mathcal{N}$ such that $\left(y_{\beta_{n}}, \beta_{n}\right) \rightarrow\left(y_{\alpha}, \alpha\right)$ and $\left(y_{\gamma_{n}}, \gamma_{n}\right) \rightarrow$ $\left(y_{\alpha}, \alpha\right)$. That is, any endpoint of a hair in $B$ is the limit of endpoints of other hairs from both above and below.

3. $B$ is a closed subset of $\mathbb{R}^{2}$.

The following facts are easily verified:

1. For any rational number $v$ and any sequence of irrationals $\alpha_{n} \in \mathcal{N}$ with $\alpha_{n} \rightarrow v$, show that the hairs $\left[y_{\alpha_{n}}, \alpha_{n}\right]$ must tend to $[\infty, v]$ in $\{\infty\} \times \mathbb{R}$.

2. Condition 2 above may be changed to: if $(y, \alpha)$ is any point in $B$ ( $y$ need not be the endpoint of the $\alpha$-hair), then there are sequences $\beta_{n} \nearrow \alpha, \gamma_{n} \searrow \alpha$ so that $\left(y_{\beta_{n}}, \beta_{n}\right) \rightarrow(y, \alpha)$ and $\left(y_{\gamma_{n}}, \gamma_{n}\right) \rightarrow(y, \alpha)$ in $B$.

3. Let $(y, \alpha) \in B$ and suppose $y$ is not the endpoint $y_{\alpha}$. Then $(y, \alpha)$ is inaccessible from $\mathbb{R}^{2} \backslash B$ in the sense that there is no continuous curve $\gamma:[0,1] \rightarrow \mathbb{R}^{2}$ such that $\gamma(t) \notin B$ for $0 \leq t<1$ and $\gamma(1)=(y, \alpha)$.

4. On the other hand, the endpoint $\left(y_{\alpha}, \alpha\right)$ is accessible from $\mathbb{R}^{2} \backslash B$. 
These facts show that a straight brush is a remarkable object from the topological point of view. Let's view a straight brush as a subset of the Riemann sphere and set $B^{*}=B \cup \infty$, i.e., the straight brush with the point at infinity added. Let $\mathcal{E}$ denote the set of endpoints of $B$, and let $\mathcal{E}^{*}=\mathcal{E} \cup \infty$. Then we have the following result, due to Mayer [May]:

Theorem 3.2. The set $\mathcal{E}^{*}$ is a connected set, but $\mathcal{E}$ is totally disconnected.

That is, the set $\mathcal{E}^{*}$ is a connected set, but if we remove just one point form this set, the resulting set is totally disconnected. Topology really is a weird subject!

The reason for this is that, if we draw the straight line in the plane $\{(t, \gamma) \mid t \in \mathbb{R}\}$ where $\gamma$ is a fixed rational, and then we adjoin the point at infinity, we find a disconnection of $\mathcal{E}$. This, however, is not a disconnection of $\mathcal{E}^{*}$. Moreover, the fact that any non-endpoint in $B$ is inaccessible shows that we cannot disconnect $\mathcal{E}^{*}$ by any other curve.

Remark. Aarts and Oversteegen have shown that any two straight brushes are ambiently homeomorphic, i.e., there is a homeomorphism of $\mathbb{R}^{2}$ taking one brush onto the other. This leads to a formal definition of a Cantor bouquet.

Definition 3.3. A Cantor bouquet is a subset of $\overline{\mathbb{C}}$ that is homeomorphic to a straight brush (with $\infty$ mapped to $\infty$ ).

Our main goal in this section is to sketch a proof of the following result:

Theorem 3.4. Suppose $0<\lambda<1 /$ e. Then $J\left(E_{\lambda}\right)$ is a Cantor bouquet.

We will construct the homeomorphism between the brush and $J\left(E_{\lambda}\right)$. To do this, we first introduce symbolic dynamics. Recall that $E_{\lambda}$ has a repelling fixed point $r_{\lambda}>0$ in $\mathbb{R}$ and that the half plane $\operatorname{Re} z<r_{\lambda}$ lies in the stable set. Similarly the horizontal strips

$$
\frac{\pi}{2}+2 k \pi<\operatorname{Im} z<\frac{\pi}{2}+(2 k+1) \pi
$$

are contained in the stable set since $E_{\lambda}$ maps these strips to $\{\operatorname{Re} z<0\}$ which is contained in $\left\{\operatorname{Re} z<r_{\lambda}\right\}$. 
We denote by $S_{k}$ the closed halfstrip given by

$$
\operatorname{Re} z \geq r_{\lambda} \text { and }-\frac{\pi}{2}+2 k \pi \leq \operatorname{Im} z \leq \frac{\pi}{2}+2 k \pi .
$$

Note that these strips contain the Julia set since the complement of the strips lies in the stable set.

Given $z \in J\left(E_{\lambda}\right)$, we define the itinerary of $z, S(z)$, as usual by

$$
S(z)=s_{0} s_{1} s_{2} \ldots
$$

where $s_{j} \in \mathbb{Z}$ and $s_{j}=k$ iff $E_{\lambda}^{j}(z) \in S_{k}$. Note that $S(z)$ is an infinite string of integers that indicates the order in which the orbit of $z$ visits the $S_{k}$. See Figure 8. We will associate to $z$ the irrational number given by the itinerary of $z$, that is, $I_{s_{0} s_{1} \ldots}$, using the decomposition of the irrationals described above. This will determine the hair in the straight brush to which $z$ is mapped. Thus we need only define the $y$-value along this hair. This takes a little work.

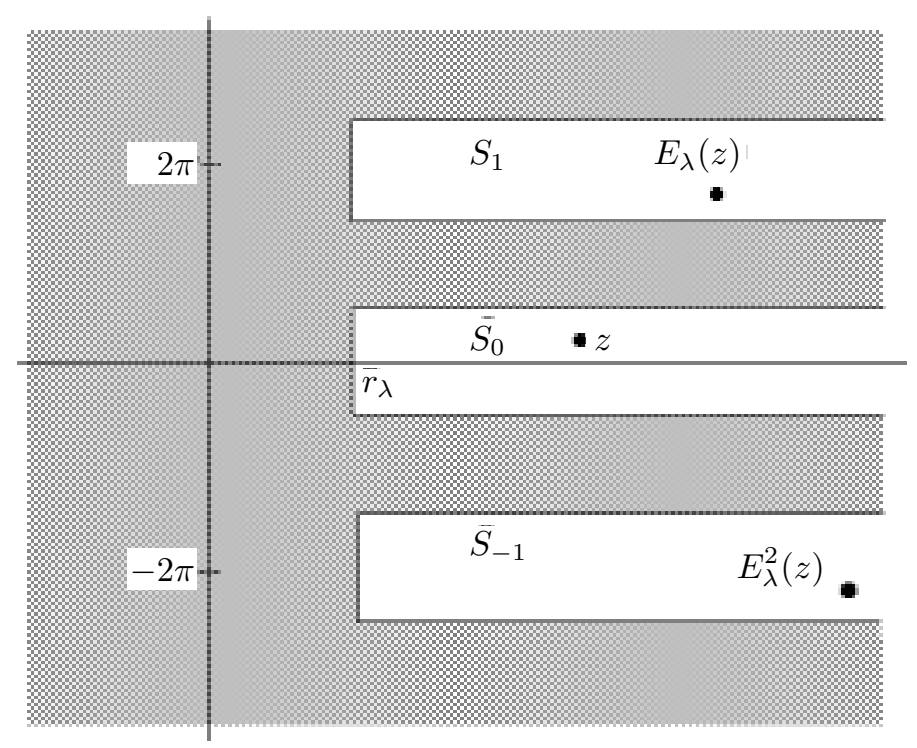

Figure 8 . The itinerary of $z$ is $0,1,-1, \ldots$. 
Given $z \in J\left(E_{\lambda}\right)$, we will construct a sequence of rectangles $R_{k}(z)$ for each $k \geq 0$. By construction, the $R_{k}(z)$ will be nested. Each $R_{k}(z)$ will have sides parallel to the axes and be contained in some strip $S_{\alpha}$. Finally each $R_{k}(z)$ will have height $\pi$. Since the $R_{k}(z)$ are nested with respect to $k$, the intersection $\bigcap_{k=0}^{\infty} R_{k}(z)$ will be a nonempty rectangle of height $\pi$ that contains $z$. We then define $h(z)$ to be the real part of the left hand edge of this limiting rectangle.

To begin the construction, we set $R_{0}\left(E_{\lambda}^{j}(z)\right)$ to be the square centered at $E_{\lambda}^{j}(z)$ with sidelength $\pi$ and contained in the strip $S_{s_{j}}$. We assume that $\operatorname{Re} w \geq 1$ in $R_{0}\left(E_{\lambda}^{j}(z)\right)$ for all $j$; otherwise we choose the rectangle

$$
R_{0}\left(E_{\lambda}^{j}(z)\right) \cap\{\operatorname{Re} z \geq 1\}
$$

for the initial box. Observe that $E_{\lambda}\left(R_{0}\left(E_{\lambda}^{j}(z)\right) \supset R_{0}\left(E_{\lambda}^{j+1}(z)\right)\right.$. Indeed, the image of $R_{0}\left(E_{\lambda}^{j}(z)\right)$ is half an annulus whose inner radius is $e^{-\pi / 2}\left|E_{\lambda}^{j+1}(z)\right|$ and outer radius $e^{\pi / 2}\left|E_{\lambda}^{j+1}(z)\right|$. Now $e^{\pi / 2}>4$ and $e^{-\pi / 2}<1 / 4$ so the image annulus is much larger than $R_{0}\left(E_{\lambda}^{j+1}\right)$. See Figure 9 .

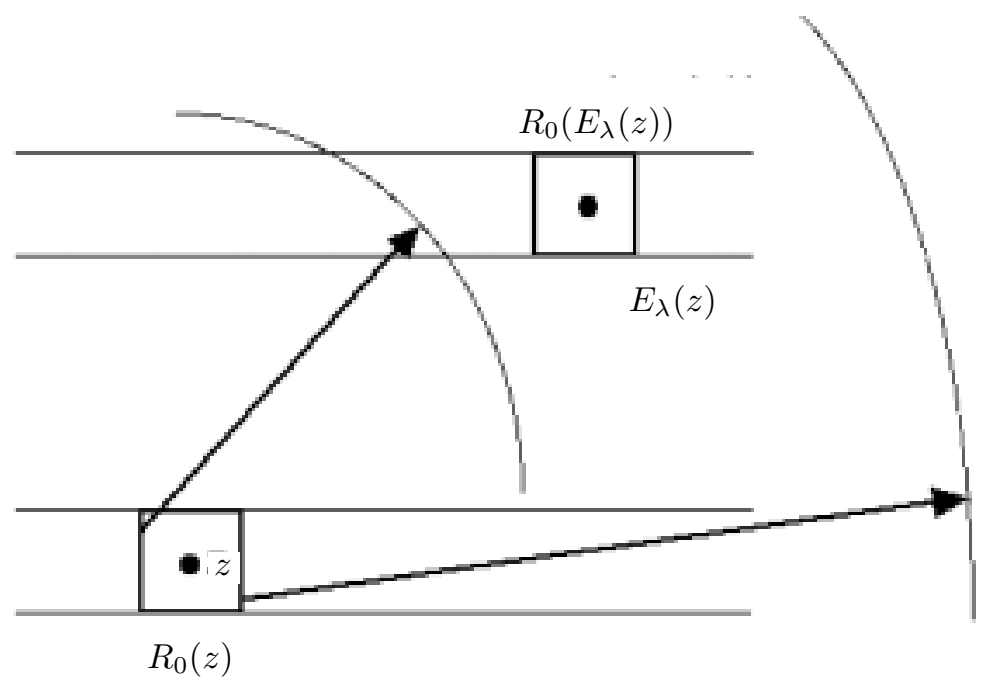

Figure 9. Construction of $R_{0}(z)$ and $R_{0}\left(E_{\lambda}\right)$.

It follows that we may find a narrower rectangle $R_{1}\left(E_{\lambda}^{j}(z)\right)$ contained in $R_{0}\left(E_{\lambda}^{j}(z)\right)$ having the property that the height of $R_{1}\left(E_{\lambda}^{j}(z)\right)$ is $\pi$ and the image $E_{\lambda}\left(R_{1}\left(E_{\lambda}^{j}(z)\right)\right)$ just covers $R_{0}\left(E_{\lambda}^{j+1}(z)\right)$. That is, $R_{1}\left(E_{\lambda}^{j}(z)\right)$ is the smallest rectangle of height $\pi$ in $R_{0}\left(E_{\lambda}^{j}(z)\right)$ whose image annulus 
is just wide enough so that $R_{0}\left(E_{\lambda}^{j+1}(z)\right)$ fits inside. See Figure 10. Note that $E_{\lambda}^{j}(z) \in R_{1}(j)$ for each $j$.

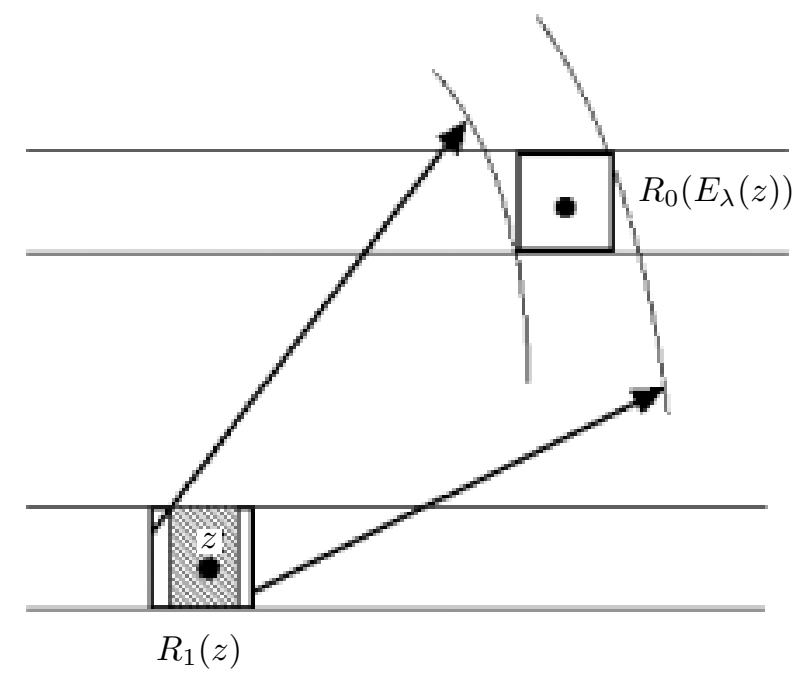

Figure 10. Construction of $R_{1}(0)$.

We now continue inductively by setting $R_{k}\left(E_{\lambda}^{j}(z)\right)$ to be the subrectangle of $R_{k-1}\left(E_{\lambda}^{j}(z)\right)$ whose image just covers $R_{k-1}\left(E_{\lambda}^{j+1}(z)\right)$. The $R_{k}\left(E_{\lambda}^{j}(z)\right)$ are clearly nested for each fixed $j$.

Example. Suppose $z=r_{\lambda}$. We have that $R_{0}(z)$ is the square bounded by $\operatorname{Re} z=r_{\lambda} \pm \pi / 2$ (or the rectangle bounded by $\operatorname{Re} z=r_{\lambda}+\pi / 2$ and $\operatorname{Re} z=1$ if $r_{\lambda}$ is close to 1 ) and $\operatorname{Im} z= \pm \pi / 2$ for each $j$. One may check that $\bigcap_{k=0}^{\infty} R_{k}(z)$ is the strip bounded by $\operatorname{Re} z=r_{\lambda}$ and $\operatorname{Re} z=\zeta$ where the circle of radius $\lambda e^{\zeta}$ passes through $\zeta \pm i \pi / 2$. See Figure 11. Hence $h\left(r_{\lambda}\right)=r_{\lambda}$.

Suppose $z$ has itinerary $S(z)=s_{0} s_{1} s_{2} \ldots$. Let $I(S(z))$ denote the irrational number determined by the sequence $S(z)$ as above. Then set $\phi(z)=(h(z), I(S(z)))$. We claim that $\phi$ is a homeomorphism onto a straight brush. For a proof, we refer to [AO]. We emphasize that, even though most hairs spiral in to their respective endpoints, the map $\phi$ is still one-to-one. 


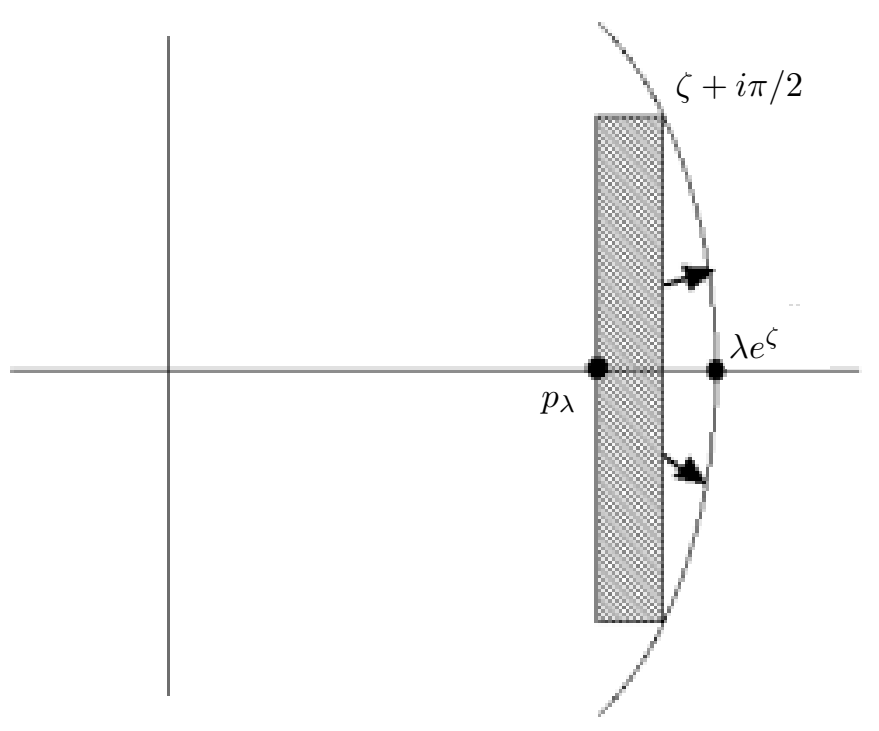

Figure 11. The intersection of $R_{j}(0)$.

\subsection{Connectedness Properties of Cantor Bouquets.}

We call the set of endpoints of a Cantor bouquet the crown. Since a Cantor bouquet is homeomorphic to a straight brush with the points at $\infty$ coinciding, it follows that any Cantor bouquet has the amazing connectedness property that the crown together with $\infty$ is connected, but the crown alone is totally disconnected.

It can be shown that the construction above works for any exponential for which there exists an attracting or neutral periodic point. See [DT]. However, in the general case, some of the hairs in the Cantor bouquet may be attached to the same point in the crown. See [BD].

McMullen [McM] has shown that the Hausdorff dimension of the Cantor bouquet constructed above is 2 but its Lebesgue measure is zero. This accounts for why Figures 2 and 3 seem to have open regions in the Julia set.

\subsection{Uniformization of the Attracting Basin.}

The basin of attraction $\Omega_{\lambda}$ of $E_{\lambda}$ is an open, dense, and simply connected subset of the Riemann sphere. Hence the Riemann Mapping Theorem guarantees the existence of a uniformization $\phi_{\lambda}: D \rightarrow \Omega_{\lambda}$. Given such a uniformization, it is natural to ask if the uniformizing map extends to the boundary of $D$. 
In order to extend $\phi_{\lambda}$ to the boundary, we need that the image of a straight ray $r e^{i \theta}$ where $\theta$ is constant under $\phi_{\lambda}$ converge to a single point as $r \rightarrow 1$. It is known that if the boundary of the uniformizing region is locally connected, then in fact $\phi_{\lambda}$ does extend continuously to $D$. On the other hand, if the boundary of the region is not locally connected, then not all rays need converge (though a full measure set of them must converge). In our case, the boundary of $\Omega_{\lambda}$ is nowhere locally connected (except at $\infty$ ). However, it is a fact that all rays do converge. In fact, they land at precisely the endpoints of the Cantor bouquet or at the point at $\infty$. This means that we can induce a map on the set of endpoints, but that map is necesarily nowhere continuous $[\mathbf{P i}]$.

In the case of a straight brush, it is clear that all rays do land at the crown of the bouquet. A direct proof for $E_{\lambda}$ is given in [DG].

In fact, it can be shown that, if we normalize the Riemann map $\phi_{\lambda}$ so that 0 is mapped to 0 , then the induced map $\phi_{\lambda}^{-} 1 \circ E_{\lambda} \circ \phi_{\lambda}$ on the unit disk is given by

$$
T_{\mu}(z)=\exp \left(i\left(\frac{\mu+\bar{\mu}}{1+z}\right)\right) .
$$

Here $\mu$ is a parameter that lies in the upper half plane and depends upon $\lambda$.

\section{Indecomposable Continua}

We now turn our attention to the case $\lambda>1 / e$. Since the orbit of 0 now tends to $\infty$, the Julia set is now the entire plane (we will actually prove this in the next section). The attracting basin for the attracting fixed point $a_{\lambda}$ disappears. What replaces it is a complicated invariant set that is an indecomposable continuum. We describe the construction of this set in this section.

Consider the horizontal strip

$$
S=\{z \mid 0 \leq \operatorname{Im} z \leq \pi\}
$$

(or its symmetric image under $z \rightarrow \bar{z}$ ). The exponential map $E_{\lambda}$ takes the boundary of $S$ to the real axis and the interior of $S$ to the upper half plane. Thus, $E_{\lambda}$ maps certain points outside of $S$ while other points remain in $S$ after one application of $E_{\lambda}$. Our goal is to investigate the set of points whose entire orbit lie in $S$. Call this set $\Lambda$. The set $\Lambda$ is clearly invariant under $E_{\lambda}$. There is a natural way to compactify this set in the plane to obtain a new set $\Gamma$. Moreover, the exponential map extends to $\Gamma$ in a natural way. Our main results in this section include: 
Theorem 4.1. $\Gamma$ is an indecomposable continuum.

Moreover, we will see that $\Lambda$ is constructed in similar fashion to a family of indecomposable continua known as Knaster continua.

As we will show in Section 4.2, the topology of $\Lambda$ is quite intricate. Despite this, we will show that the dynamics of $E_{\lambda}$ on $\Lambda$ is quite tame. Specifically, we will prove:

Theorem 4.2. The restriction of $E_{\lambda}$ to $\Lambda-\{$ orbit of 0$\}$ is a homeomorphism. This map has a unique repelling fixed point $w_{\lambda} \in \Lambda$, and the $\alpha$-limit set of all points in $\Lambda$ is $w_{\lambda}$. On the other hand, if $z \in \Lambda, z \neq w_{\lambda}$, then the $\omega$-limit set of $z$ is either

1. The point at $\infty$, or

2. The orbit of 0 under $E_{\lambda}$ together with the point at $\infty$.

Thus we see that $E_{\lambda}$ possesses an interesting mixture of topology and dynamics in the case where the Julia set is the whole plane. In the plane the dynamics of $E_{\lambda}$ are quite chaotic, but the overall topology is tame. On our invariant set $\Lambda$, however, it is the topology that is rich, but the dynamics are tame.

\subsection{Topological Preliminaries.}

In this section we review some of the basic topological ideas associated with indecomposable continua. See $[\mathbf{K u}]$ for a more extensive introduction to these concepts.

Recall that a continuum is a compact, connected space. A continuum is decomposable if it is the union of two proper subcontinua. Otherwise, it is indecomposable. One famous example of an indecomposable continuum is the Knaster continuum, K. One way to construct this set is to begin with the Cantor middle-thirds set. Then draw the semi-circles lying in the upper half plane with center at $(1 / 2,0)$ that connect each pair of points in the Cantor set that are equidistant from $1 / 2$. Next draw all semicircles in the lower half plane which have for each $n \geq 1$ centers at $\left(5 /\left(2 \cdot 3^{n}\right), 0\right)$ and pass through each point in the Cantor set lying in the interval

$$
2 / 3^{n} \leq x \leq 1 / 3^{n-1}
$$

The resulting set is partially depicted in Figure 12 . 


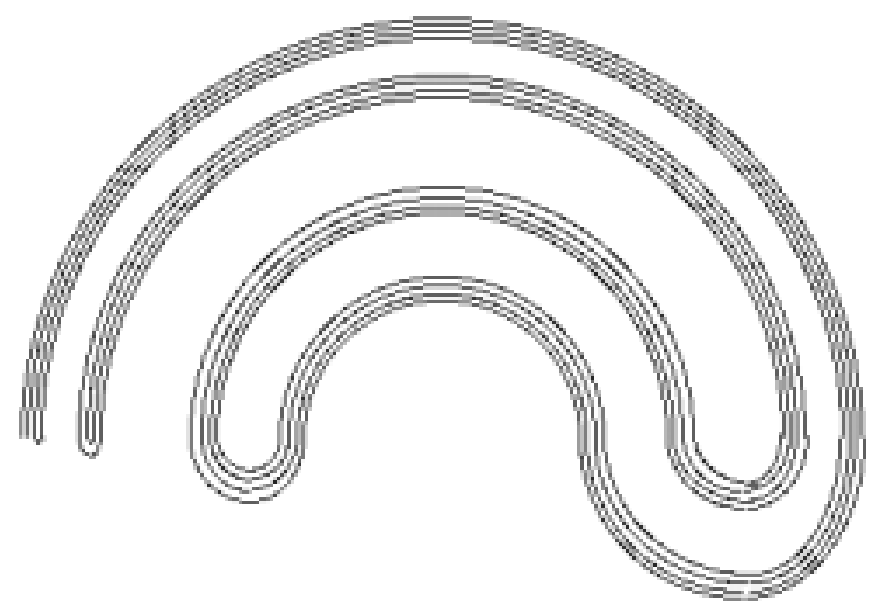

Figure 12. The Knaster Continuum.

For a proof that this set is indecomposable, we refer to $[\mathbf{K u}]$. Dynamically, this set appears as the closure of the unstable manifold of Smale's horseshoe map (see $[\mathbf{B a}],[\mathbf{S m}]$ ).

Note that the curve passing through the origin in this set is dense, since it passes through each of the endpoints of the Cantor set. It also accumulates everywhere upon itself. Such a phenomenon gives a criterion for a continuum to be indecomposable, as was shown by S. Curry.

Theorem 4.3. Suppose $X$ is a one-dimensional nonseparating plane continuum which is the closure of a ray that limits upon itself. Then $X$ is indecomposable.

We refer to $[\mathbf{C u}]$ for a proof.

Another view of the Knaster continuum which is intimately related to our own construction is as follows. Begin with the unit square $S_{0}$ in the plane. Next remove a "canal" $C_{1}$ from $S_{0}$ whose boundary lies within a distance $1 / 3$ of each boundary point of $S_{0}$ as depicted in Figure 2. Call this set $S_{1}$. Next remove a new canal $C_{2}$ from $S_{1}$. This time the boundary of $C_{2}$ should be within $1 / 9$ of the boundary of $S_{1}$ as depicted in Figure 13. It is possible to continue this construction inductively in such a way that the resulting set is homeomorphic to the Knaster continuum. 

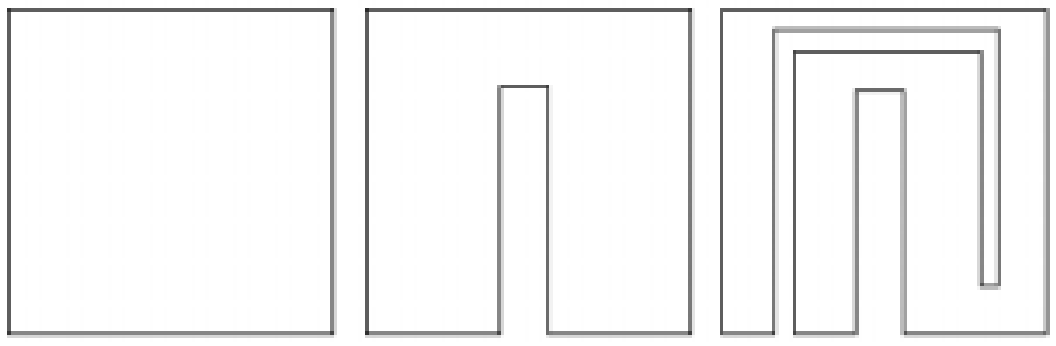

Figure 13. A different construction of the Knaster Continuum.

\subsection{Construction of $\Lambda$.}

Recall that the strip $S$ is given by $\{z \mid 0 \leq \operatorname{Im}(z) \leq \pi\}$. Note that $E_{\lambda}$ maps $S$ in one-to-one fashion onto $\{z \mid \operatorname{Im} z \geq 0\}-\{0\}$. Hence a branch of $E_{\lambda}^{-1}$ is well defined taking $S-\{0\}$ to $S$. In fact, $E_{\lambda}^{-n}$ is defined for all $n$ on $S-\{$ orbit of 0$\}$. We will always assume that $E_{\lambda}^{-n}$ means $E_{\lambda}^{-n}$ restricted to this subset of $S$.

Define

$$
\Lambda=\left\{z \mid E_{\lambda}^{n}(z) \in S \text { for all } n \geq 0\right\}
$$

If $z \in \Lambda$ it follows immediately that $E_{\lambda}^{n}(z) \in S$ for all $n \in \mathbf{Z}$ provided $z$ does not lie on the forward orbit of 0 . Our goal is to understand the structure of $\Lambda$.

Toward that end we define $L_{n}$ to be the set of points in $S$ that leave $S$ at precisely the $n^{\text {th }}$ iteration of $E_{\lambda}$. That is,

$$
L_{n}=\left\{z \in S \mid E_{\lambda}^{i}(z) \in S \text { for } 0 \leq i<n \text { but } E_{\lambda}^{n}(z) \notin S\right\}
$$

Let $B_{n}$ be the boundary of $L_{n}$.

Recall that $E_{\lambda}$ maps a vertical segment in $S$ to a semi-circle in the upper half plane centered at 0 with endpoints in $\mathbb{R}$. Either this semicircle is completely contained in $S$ or else an open arc lies outside $S$. As a consequence, $L_{1}$ is an open simply connected region which extends to $\infty$ toward the right in $S$ as shown in Figure 14 . There is a natural parametrization $\gamma_{1}: \mathbb{R} \rightarrow B_{1}$ defined by

$$
E_{\lambda}\left(\gamma_{1}(t)\right)=t+i \pi
$$

As a consequence,

$$
\lim _{t \rightarrow \pm \infty} \operatorname{Re} \gamma_{1}(t)=\infty
$$




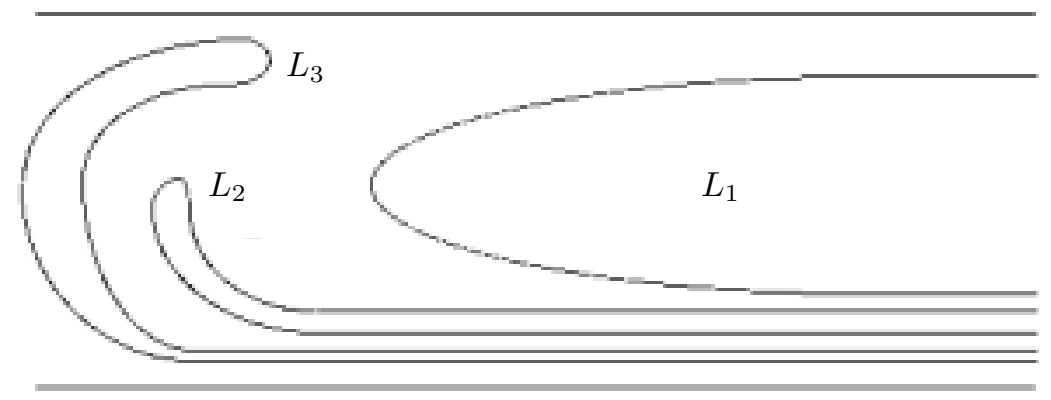

Figure 14. Construction of the $L_{n}$.

If $c>0$ is large, the segment $\operatorname{Re} z=c$ in $S$ meets $S-L_{1}$ in two vertical segments $v_{+}$and $v_{-}$with $\operatorname{Im} v_{-}>\operatorname{Im} v_{+}$. $E_{\lambda}$ maps $v_{-}$to an arc of a circle in $S \cap\{z \mid \operatorname{Re} z<0\}$ while $E_{\lambda}$ maps $v_{+}$to an arc of a circle in $S \cap\{z \mid \operatorname{Re} z>0\}$. As a consequence, if $c$ is large, $v_{+}$meets $L_{2}$ in an open interval. Since $L_{2}=E_{\lambda}^{-1}\left(L_{1}\right)$, it follows that $L_{2}$ is an open simply connected subset of $S$ that extends to $\infty$ in the right half plane below $L_{1}$.

Continuing inductively, we see that $L_{n}$ is an open, simply connected subset of $S$ that extends to $\infty$ toward the right in $S$. We may also parametrize the boundary $B_{n}$ of $L_{n}$ by $\gamma_{n}: \mathbf{R} \rightarrow B_{n}$ where

$$
E_{\lambda}^{n}\left(\gamma_{n}(t)\right)=t+i \pi
$$

as before. Again

$$
\lim _{t \rightarrow \pm \infty} \operatorname{Re} \gamma_{n}(t)=\infty
$$

Since each $L_{n}$ is open, it follows that $\Lambda$ is a closed subset of $S$.

Proposition 4.4. The set $\bigcup_{i=n}^{\infty} B_{i}$ is dense in $\Lambda$ for each $n>0$.

Proof: Let $z \in \Lambda$ and suppose $z \notin B_{i}$ for any $i$. Let $U$ be an open connected neighborhood of $z$. Fix $n>0$. Since $E_{\lambda}^{i}(z) \in S$ for all $i$, we may choose a connected neighborhood $V \subset U$ of $z$ such that $E_{\lambda}^{i}(V) \subset S$ for $i=0, \ldots, n$.

Now the family of functions $\left\{E_{\lambda}^{i}\right\}$ is not normal on $V$, since $z$ belongs to the Julia set of $E_{\lambda}$. Consequently, $\bigcup_{i=0}^{\infty} E_{\lambda}^{i}(V)$ covers $\mathbb{C}-\{0\}$. In particular, there is $m>n$ such that $E_{\lambda}^{m}(V)$ meets the exterior of $S$. Since $E_{\lambda}^{m}(z) \in S$, it follows that $E_{\lambda}^{m}(V)$ meets the boundary of $S$. Applying $E_{\lambda}^{-m}$, we see that $B_{m}$ meets $V$. 
In fact, it follows that for any $z \in \Lambda$ and any neighborhood $U$ of $z$, all but finitely many of the $B_{m}$ meet $V$. This follows from the fact that $E_{\lambda}$ has fixed points outside of $S$ (in fact one such point in each horizontal strip of width $2 \pi$ - see $[\mathbf{D K}])$, so we may assume that $E_{\lambda}^{m}(V)$ contains this fixed point for all sufficiently large $m$. In particular, we have shown:

Proposition 4.5. Let $z \in \Lambda$ and suppose that $V$ is any connected neighborhood of $z$. Then $E_{\lambda}^{m}(V)$ meets the boundary of $S$ for all sufficiently large $m$.

Proposition 4.6. $\Lambda$ is a connected subset of $S$.

Proof: Let $G$ be the union of the boundaries of the $L_{i}$ for all $i$. Since $\Lambda$ is the closure of $G$, it suffices to show that $G$ is connected. Suppose that this is not true. Then we can write $G$ as the union of two disjoint sets $A$ and $B$. One of $A$ or $B$ must contain infinitely many of the boundaries of the $L_{i}$. Say $A$ does. But then, if $b \in B$, the previous proposition guarantees that infinitely many of these boundaries meet any neighborhood of $b$. Hence $b$ belongs to the closure of $A$. This contradiction establishes the result.

We can now prove:

Theorem 4.7. There is a natural compactification $\Gamma$ of $\Lambda$ that makes $\Gamma$ into an indecomposable continuum.

Proof: We first compactify $\Lambda$ by adjoining the backward orbit of 0 . To do this we identify the "points" $(-\infty, 0)$ and $(-\infty, \pi)$ in $S$ : this gives $E_{\lambda}^{-1}(0)$. We then identify the points $(\infty, \pi)$ and $\lim _{t \rightarrow-\infty} \gamma_{1}(t)$. This gives $E_{\lambda}^{-2}(0)$. For each $n>1$ we identify

$$
\lim _{t \rightarrow \infty} \gamma_{n}(t)
$$

and

$$
\lim _{t \rightarrow-\infty} \gamma_{n+1}(t)
$$

to yield $E_{\lambda}^{-n-1}(0)$. This augmented space $\Gamma$ may easily be embedded in the plane. See Figure 15. Moreover, if we extend the $B_{i}$ and the lines $y=0$ and $y=\pi$ in the natural way to include these new points, then this yields a curve which accumulates everywhere on itself but does not separate the plane. See the proposition above. By a theorem of S. Curry $[\mathbf{C u}]$, it follows that $\Gamma$ is indecomposable. 


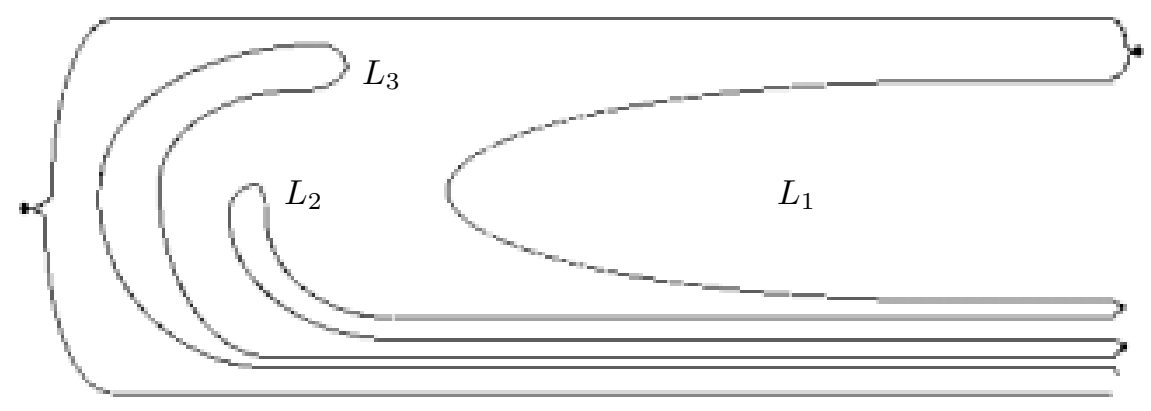

Figure 15. Embedding $\Gamma$ in the plane.

As a consequence of this theorem, $\Lambda$ must contain uncountably many composants (see $[\mathbf{K u}$, p. 213]). In fact, in $[\mathbf{D K}]$ it is shown that $\Lambda$ contains uncountably many curves.

\subsection{Dynamics on $\Lambda$.}

In this section we describe completely the dynamics of $E_{\lambda}$ on $\Lambda$.

Proposition 4.8. There exists a unique fixed point $w_{\lambda}$ in $S$ if $\lambda>1 /$ e. Moreover, $w_{\lambda}$ is repelling and, if $z \in S-$ orbit of $0, E_{\lambda}^{-n}(z) \rightarrow$ $w_{\lambda}$ as $n \rightarrow \infty$.

Proof: First consider the equation

$$
\lambda e^{y \cot y} \sin y=y
$$

Since $y \cot y \rightarrow 1$ as $y \rightarrow 0$ and $\lambda e>1$, we have $\lambda e^{y \cot y} \sin y>y$ for $y$ small and positive. Since the left-hand side of this equation vanishes when $y=\pi$, it follows that this equation has at least one solution $y_{\lambda}$ in the interval $0<y<\pi$.

Let $x_{\lambda}=y_{\lambda} \cot y_{\lambda}$. Then one may easily check that $w_{\lambda}=x_{\lambda}+i y_{\lambda}$ is a fixed point for $E_{\lambda}$ in the interior of $S$. Since the interior of $S$ is conformally equivalent to a disk and $E_{\lambda}^{-1}$ is holomorphic, it follows from the Schwarz Lemma that $w_{\lambda}$ is an attracting fixed point for the restriction of $E_{\lambda}^{-1}$ to $S$ and that $E_{\lambda}^{-n}(z) \rightarrow w_{\lambda}$ for all $z \in S$. 


\section{Remarks.}

1. Thus the $\alpha$-limit set of any point in $\Lambda$ is $w_{\lambda}$.

2. The bound $\lambda>1 / e$ is necessary for this result, since we know that $E_{\lambda}$ has two fixed points on the real axis for any positive $\lambda<1 / e$. These fixed points coalesce at 1 as $\lambda \rightarrow 1 / e$ and then separate into a pair of conjugate fixed points, one of which lies in $S$.

We now describe the $\omega$-limit set of any point in $\Lambda$. Clearly, if $z \in B_{n}$ then $E_{\lambda}^{n+1}(z) \in \mathbb{R}$ and so the $\omega$-limit set of $z$ is infinity. Thus we need only consider points in $\Lambda$ that do not lie in $B_{n}$. We will show:

Theorem 4.9. Suppose $z \in \Lambda$ and $z \neq w_{\lambda}, z \notin B_{n}$ for any $n$. Then the $\omega$-limit set of $z$ is the orbit of 0 under $E_{\lambda}$ together with the point at infinity.

To prove this we first need a lemma.

Lemma 4.10. Suppose $z \in \Lambda, z \neq w_{\lambda}$. Then $E_{\lambda}^{n}(z)$ approaches the boundary of $S$ as $n \rightarrow \infty$.

Proof: Let $h$ be the uniformization of the interior of $S$ taking $S$ to the open unit disk and $w_{\lambda}$ to 0 . Recall that $E_{\lambda}^{-1}$ is well defined on $S$ and takes $S$ inside itself. Then $g=h \circ E_{\lambda}^{-1} \circ h^{-1}$ is an analytic map of the open disk into itself with a fixed point at 0 . This fixed point is therefore attracting by the Schwarz Lemma. Moreover, if $|z|>0$ we have $|g(z)|<|z|$. As a consequence, if $\left\{z_{n}\right\}$ is an orbit in $\Lambda$, we have $\left|h\left(z_{n+1}\right)\right|>\left|h\left(z_{n}\right)\right|$, and so $\left|h\left(z_{n}\right)\right| \rightarrow 1$ as $n \rightarrow \infty$.

The remainder of the proof is essentially contained in [DK] (see pp. 4549). In that paper it is shown that there is a "quadrilateral" $Q$ containing a neighborhood of 0 in $\mathbb{R}$ as depicted in Figure 5 . The set $Q$ has the following properties:

1. If $z \in \Lambda-\bigcup_{n} B_{n}$ and $z \neq w_{\lambda}$, then the forward orbit of $z$ meets $Q$ infinitely often.

2. $Q$ contains infinitely many closed "rectangles" $R_{k}, R_{k+1}, R_{k+2}, \ldots$ for some $k>1$ having the property that if $z \in R_{j}$, then $E_{\lambda}^{j}(z) \in Q$ but $E_{\lambda}^{i}(z) \notin Q$ for $0<i<j$.

3. If $z \notin \bigcup_{j=k}^{\infty} R_{j}$, then $z \in L_{n}$ for some $n$.

4. $E_{\lambda}^{j}\left(R_{j}\right)$ is a "horseshoe" shaped region lying below $R_{j}$ in $Q$ as depicted in Figure 5.

5. $\lim _{j \rightarrow \infty} E_{\lambda}^{j}\left(R_{j}\right)=\{0\}$. 


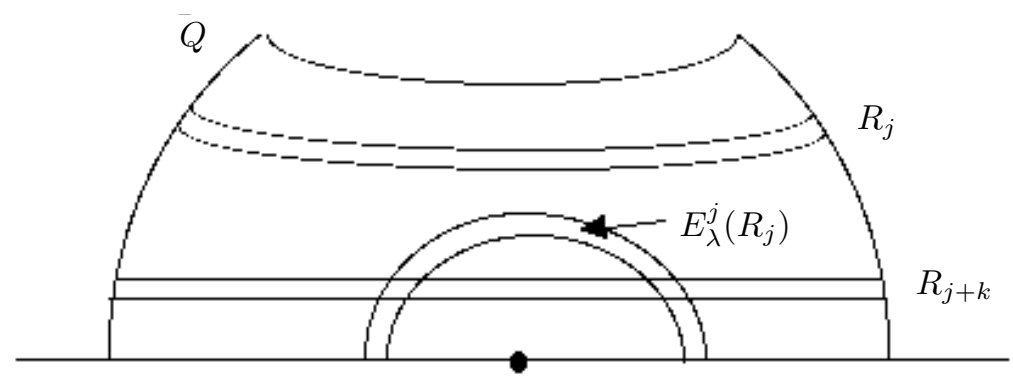

Figure 16. The return map on $Q$.

As a consequence of these facts, any point in $\Lambda$ has orbit that meets the $\cup R_{j}$ infinitely often. We may thus define a return map

$$
\Phi: \Lambda \cap\left(\cup_{j} R_{j}\right) \rightarrow \Lambda \cap\left(\cup_{j} R_{j}\right)
$$

by

$$
\Phi(z)=E_{\lambda}^{j}(z)
$$

if $z \in R_{j}$. By item $4, \Phi(z)$ lies in some $R_{k}$ with $k>j$. By item 5 , it follows that

$$
\Phi^{n}(z) \rightarrow 0
$$

for any $z \in \Lambda \cap Q$. Consequently, the $\omega$-limit set of $z$ contains the orbit of 0 and infinity.

For the opposite containment, suppose that the forward orbit of $z$ accumulates on a point $q$. By the Lemma, $q$ lies in the boundary of $S$. Now the orbit of $q$ must also accumulate on the preimages of $q$. If $q$ does not lie on the orbit of 0 , then these preimages form an infinite set, and some points in this set lie on the boundaries of the $L_{n}$. But these points lie in the interior of $S$, and this contradicts the Lemma. Thus the orbit of $z$ can only accumulate in the finite plane on points on the orbit of 0 . Since the "preimage" of 0 is infinity, the orbit also accumulates at infinity.

It is known that there are uncountably many curves in the $\lambda$-plane having the property that, if $\lambda$ lies on one of these curves, then $E_{\lambda}^{n}(0) \rightarrow \infty$. Consequently, for such a $\lambda$-value, the Julia set of $E_{\lambda}$ is again the complex plane. For these $\lambda$-values, a variant of the above construction also yields invariant indecomposable continua in the Julia set. Whether these continua are homeomorphic to any of those constructed above is an open question. We plan to discuss these constructions in a later paper. 
Douady and Goldberg [DoG] have shown that if $\lambda, \mu>1 / e$, then $E_{\lambda}$ and $E_{\mu}$ are not topologically conjugate. Each such map possesses invariant indecomposable continua $\Lambda_{\lambda}$ and $\Lambda_{\mu}$ in $S$, and the dynamics on each are similar, as shown above. In fact, one can show that each pair of these invariant sets is non-homeomorphic.

As a final remark, M. Lyubich has shown that each $\Lambda_{\lambda}$ is a set of measure 0 in $S$. Indeed, it follows from his work $[\mathbf{L y}]$ that the set of points in $\mathbb{C}$ whose orbits have arguments that are equidistributed on the unit circle have full measure. In $\Lambda_{\lambda}$, the arguments of all orbits tend to 0 and/or $\pi$, and so $\Lambda_{\lambda}$ has measure 0 in $S$.

\section{After the Explosion}

As we have mentioned, when $\lambda>1 / e$, the Julia set of $E_{\lambda}$ is the entire plane. In 1981, Misiurewicz showed that $J\left(E_{1}\right)=\mathbb{C}$, answering a sixty-year-old question of Fatou. We present his proof of this fact below, generalizing it to the case $\lambda>1 / e$.

The following proposition highlights one of the differences between $E_{\lambda}(z)$ and polynomials: points which tend to $\infty$ under iteration of $E_{\lambda}$ need not be in the stable set.

Proposition 5.1. The real line is contained in $J\left(E_{\lambda}\right)$ and hence all preimages of the real line lie in $J\left(E_{\lambda}\right)$.

Proof: Let $S$ denote the strip $|\operatorname{Im}(z)| \leq \pi$. Suppose $E_{\lambda}^{j}(z) \in \mathbb{R}$. Hence $E_{\lambda}^{n}(z) \rightarrow \infty$. Let $U$ be a neighborhood of $z$. Then $E_{\lambda}^{i}(U)$ meets the real line for all sufficiently large $i$. Drawing on the results of the previous section, there are points arbitrarily close to $E_{\lambda}^{i}(z)$ whose images eventually lie in the far left half plane, and so their next images lie in the unit disk about 0 . Thus the family of iterates $\left\{E_{\lambda}^{n}\right\}$ is not a normal family on $U$ and so $z \in J\left(E_{\lambda}\right)$.

Thus to show that $J\left(E_{\lambda}\right)=\mathbb{C}$, it suffices to show that inverse images of the real line are dense in $\mathbb{C}$. For this, we need several lemmas.

Lemma 5.2. $\left|\operatorname{Im}\left(E_{\lambda}^{n}(z)\right)\right| \leq\left|\left(E_{\lambda}^{n}\right)^{\prime}(z)\right|$

Proof: If $z \in \mathbb{R}$ the inequality is trivial. Hence we assume $z \notin \mathbb{R}$. If $z=x+i y$, we have

$$
\begin{aligned}
\left|\operatorname{Im}\left(E_{\lambda}(z)\right)\right| & =\lambda e^{x}|\sin y| \\
& \leq \lambda e^{x}|y| \\
& =\left|E_{\lambda}^{\prime}(z)\right||\operatorname{Im}(z)|
\end{aligned}
$$


so that

$$
\frac{\left|\operatorname{Im}\left(E_{\lambda}(z)\right)\right|}{|\operatorname{Im}(z)|} \leq\left|E_{\lambda}^{\prime}(z)\right|
$$

since $z \notin \mathbb{R}$. More generally, if $E_{\lambda}^{n}(z) \notin \mathbb{R}$, we may apply this inequality repeatedly to find

$$
\begin{aligned}
\frac{\left|\operatorname{Im}\left(E_{\lambda}^{n}(z)\right)\right|}{\left|\operatorname{Im}\left(E_{\lambda}(z)\right)\right|} & =\prod_{i=1}^{n-1} \frac{\left|\operatorname{Im} E_{\lambda}\left(E_{\lambda}^{i}(z)\right)\right|}{\left|\operatorname{Im}\left(E_{\lambda}^{i}(z)\right)\right|} \\
& \leq \prod_{i=1}^{n-1}\left|E_{\lambda}^{\prime}\left(E_{\lambda}^{i}(z)\right)\right| .
\end{aligned}
$$

Since $\left|\operatorname{Im}\left(E_{\lambda}(z)\right)\right| \leq\left|E_{\lambda}(z)\right|=\left|E_{\lambda}^{\prime}(z)\right|$ we may write

$$
\begin{aligned}
\left|\operatorname{Im}\left(E_{\lambda}^{n}(z)\right)\right| & \leq \prod_{i=0}^{n-1}\left|E_{\lambda}^{\prime}\left(E_{\lambda}^{i}(z)\right)\right| \\
& =\left|\left(E_{\lambda}^{n}\right)^{\prime}(z)\right| .
\end{aligned}
$$

Now let $W=\{z|| \operatorname{Im} z \mid \leq \pi / 3\}$. We have seen that the orbits of an open set of points in $W \subset S$ leave $S$ and hence $W$ under iteration. The next lemma shows, however, that the orbits of most of these points must eventually return.

Lemma 5.3. Let $U$ be an open connected set. Then only finitely many of the $E_{\lambda}^{n}(U)$ can be disjoint from $W$.

Proof: Let us assume that infinitely many of the images of $U$ are disjoint from $W$. If there is an $n$ for which $E_{\lambda}^{n}$ is not a homeomorphism taking $U$ onto its image, then there exist $z_{1}, z_{2} \in U, z_{1} \neq z_{2}$, for which $E_{\lambda}^{n}\left(z_{1}\right)=E_{\lambda}^{n}\left(z_{2}\right)$. Consequently, there is a $j$ for which $E_{\lambda}^{j}\left(z_{1}\right)=E_{\lambda}^{j}\left(z_{2}\right)+2 k \pi i$ for some $k \in \mathbf{Z}-\{0\}$. But then $E_{\lambda}^{j}(U)$ must meet a horizontal line of the form $y=2 m \pi$ for $m \in \mathbf{Z}$ and so $E_{\lambda}^{j+1}(U)$ meets $\mathbb{R}$. Hence $E_{\lambda}^{j+\alpha}(U)$ meets $\mathbb{R}$ for all $\alpha>0$ and only finitely many of the images of $U$ can be disjoint from $W$. We thus conclude that each $E_{\lambda}^{n}$ must be a homeomorphism on $U$.

Now suppose there is a sequence $n_{j}$ such that for each $j, E_{\lambda}^{n_{j}}(U) \cap W=$ $\phi$. By the previous lemma, $\left|\left(E_{\lambda}^{n_{j}}\right)^{\prime}(z)\right| \geq(\pi / 3)^{n_{j}}$ for each $j$ and all $z \in U$. It follows that, if $U$ contains a disk of radius $\delta>0$, then $E_{\lambda}^{n_{j}}(U)$ contains a disk of radius $\delta(\pi / 3)^{n_{j}}$. Hence for $j$ large enough, $E_{\lambda}^{n_{j}}(U)$ must meet a line of the form $y=2 \pi$ and again we are done.

We can now prove 
Theorem 5.4. $J\left(E_{\lambda}\right)=\mathbb{C}$.

Proof: By Proposition 5.1, it suffices to show that any open set in $\mathbb{C}$ contains some preimage of $\mathbb{R}$. To that end, let $U$ be open and connected and suppose $E^{n}(U) \cap \mathbb{R}=\emptyset$ for each $n$. By Montel's Theorem, $\left\{E_{\lambda}^{n}\right\}$ is a normal family on $U$.

By the previous lemma, we have that at most finitely many iterates of $U$ are disjoint from $W$. Since none of the iterates of $U$ meet the boundary of $S$, it follows that all but finitely many of the iterates of $U$ lie in $S$. By replacing $U$ by $E_{\lambda}^{n}(U)$, we may assume that all of the iterates of $U$ lie in $S$.

Now we invoke the results of the previous section. The $\omega$-limit set of any point in $U$ must be the orbit of 0 and $\infty$. Hence the orbit of $U$ must enter any small neighborhood of 0 infinitely often. But we saw above that, after entering this neighborhood, subsequent iterates of $U$ move along the real axis until suddenly jumping above the exit set $L_{1}$. But this image lies outside the strip $W$. Since this happens infinitely often, we have infinitely many images of $U$ that do not meet $W$. This contradiction establishes the theorem.

\section{References}

[A] P. Atela, Bifurcations of dynamic rays in complex polynomials of degree two, Ergodic Theory Dynam. Systems 12 (1991), 401-423.

[AO] J. Aarts And L. Oversteegen, The geometry of Julia sets, Trans. Amer. Math. Soc. 338 (1993), 897-918.

[B] B. Branner, The Mandelbrot Set, in "Chaos and Fractals: The Mathematics Behind the Computer Graphics," Amer. Math. Soc., 1989, pp. 75-106.

[Ba] M. Barge, Horseshoe maps and inverse limits, Pacific J. Math. 121 (1986), 29-39.

[Ba1] I. N. BAKER, Wandering domains in the iteration of entire functions, Proc. London Math. Soc. 49 (1984), 563-576.

[Ba2] I. N. BAKER, Repulsive fixpoints of entire functions, Math. Z. 104 (1968), 252-256.

[BD] R. Bhattacharjee R. L. Devaney, Tying hairs for structurally stable exponentials, Preprint.

[Bl] P. Blanchard, Complex analytic dynamics on the Riemann sphere, Bull. Amer. Math. Soc. 2 (1984), 85-141. 
[Bo] C. Bodelon et AL., Hairs for the complex exponential family, Preprint.

[BR] I. N. BAKER AND P. RIPPON, Iteration of exponential functions, Ann. Acad. Sci. Fenn. Ser. A I Math. 9 (1984), 49-77.

$[\mathrm{Cu}]$ S. CURRY, One-dimensional nonseparating plane continua with disjoint $\epsilon$-dense subcontinua, Topology Appl. 39 (1991), 145-151.

[D] R. L. Devaney, The structural instability of $\exp (z)$, Proc. Amer. Math. Soc. 94 (1985), 545-548.

[DG] R. L. Devaney and L. Goldberg, Uniformization of attracting basins for exponential maps, Duke Math. J. 55 (1987), 253-266.

[DH] A. Douady and J. Hubbard, Étude dynamique des polynômes complexes, Publ. Math. Orsay 4, Université de Paris-Sud (1985), $154 \mathrm{pp}$.

[DH1] A. Douady And J. Hubbard, Itération des polynômes quadratiques complexes, C. R. Acad. Sci. Paris Sér. I Math. 29 (1982), $123-126$.

[DK] R. L. Devaney and M. KRych, Dynamics of $\exp (z)$, Ergodic Theory Dynam. Systems 4 (1984), 35-52.

[DoG] A. Doundy and L. Goldberg, The nonconjugacy of certain exponential functions, in "Holomorphic Functions and Moduli," MSRI Publ. Springer Verlag, 1988, pp. 1-8.

[DT] R. L. Devaney and F. Tangerman, Dynamics of entire functions near the essential singularity, Ergodic Theory Dynam. Systems 6 (1986), 489-503.

[EL] A. Eremenko and M. Yu. Lyubich, Iterates of entire functions, Dokl. Akad. Nauk SSSR 279 (1984), 25-27; English translation in Soviet Math. Dokl. 30 (1984), 592-594.

[GK] L. R. Goldberg and L. Keen, A finiteness theorem for a dynamical class of entire functions, Ergodic Theory Dynam. Systems 6 (1986), 183-192.

[Ku] K. Kuratowski, "Topology," Vol. 2, Academic Press, New York, 1968.

[Ly] M. Lyubich, Measurable dynamics of the exponential, Soviet Math. Dokl. 35 (1987), 223-226.

[Ma] J. MAYER, An explosion point for the set of endpoints of the Julia set of $\lambda \exp (z)$, Ergodic Theory Dynam. Systems 10 (1990), $177-184$.

[McM] C. McMullen, Area and Hausdorff dimension of Julia sets of entire functions, Trans. Amer. Math. Soc. 300 (1987), 329-342. 
[Mi] M. Misiurewicz, On iterates of $e^{z}$, Ergodic Theory Dynam. Systems 1 (1981), 103-106.

[Pi] G. Piranian, The boundary of a simply connected domain, Bull. Amer. Math. Soc. 64 (1958), 45-55.

[Sm] S. Smale, Diffeomorphisms with many periodic points, in "Differential and Combinatorial Topology," Princeton University Press, 1964, pp. 63-80.

[Su] D. Sullivan, Quasiconformal maps and dynamical systems I, Solutions of the Fatou-Julia problem on wandering domains, Ann. of Math. 122 (1985), 401-418.

\author{
Department of Mathematics \\ Boston University \\ 111 Cummington Street \\ Boston MA 02215 \\ U.S.A. \\ e-mail: bob@bu.edu.
}

Primera versió rebuda el 18 de desembre de 1998 , darrera versió rebuda el 26 de març de 1999 